\title{
Application of 3D technology and printing for femoral derotation osteotomy: case and technical report
}

\author{
Nicolás Fiz ${ }^{1}$, Diego Delgado², Xabier Sánchez ${ }^{3}$, Pello Sánchez ${ }^{2}$, Ane Miren Bilbao ${ }^{1}$, Jaime Oraa ${ }^{1}$, Mikel \\ Sánchez ${ }^{1,2}$ \\ ${ }^{1}$ Arthroscopy Surgery Unit, ${ }^{2}$ Advanced Biological Therapy Unit, Hospital Vithas San José, Vitoria-Gasteiz, Spain; ${ }^{3}$ Mizar Additive Manufacturing, \\ Vitoria-Gasteiz, Spain \\ Correspondence to: Dr. Mikel Sánchez. Arthroscopic Surgery Unit, Hospital Vithas San Jose, Beato Tomás de Zumarraga 10, 01008, Vitoria, Spain. \\ Email: mikel.sanchez@ucatrauma.com.
}

\begin{abstract}
In some surgical techniques like femoral derotation osteotomy, accuracy is a key factor that often is not optimal because of the lack of appropriate technology. 3D printing is emerging in many professional areas and its use in the medical field may enhance the results of certain surgeries. This case describes a patient who underwent an intramedullary nail fixation to treat a femoral shaft fracture. After nine months, the patient presented hip pain and "in toe" walking caused by a malrotation produced during the surgery. To address the consequent femoral derotation osteotomy, 3D technology was used throughout the whole process. A 3D model of the patient's femur was created to conduct a real and accuracy assessment of femoral anteversion. Then, a customized surgical guide was designed and printed to ensure the proper alignment during surgery. Given the success of this surgery, 3D printing can be considered a quick and inexpensive tool to improve surgical results.
\end{abstract}

Keywords: 3D printing; 3D technology; femoral derotation osteotomy; femoral malrotation; femoral anteversion; intramedullary nail fixation

Submitted Jun 19, 2017. Accepted for publication Jul 01, 2017.

doi: $10.21037 / \mathrm{atm} .2017 .07 .03$

View this article at: http://dx.doi.org/10.21037/atm.2017.07.03

\section{Introduction}

The lack of suitable technology that ensures accuracy in some surgical techniques makes the final results unsuccessful and negative for the patient. The emergence of $3 \mathrm{D}$ technology in medical field can help the surgeon to improve their surgical techniques as in the femoral derotation osteotomy described below.

\section{Case presentation}

The patient was a healthy 22 -year-old woman who was hospitalized after suffering a high-energy trauma that affected her left leg. The clinical and radiological examination revealed a closed fracture in the middle third of the femoral shaft. Twenty-four hours after admission, patient underwent surgery. An intramedullary nailing fixation was performed, using a T2 femoral nailing system with distal and proximal locking (Striker, USA). The patient was discharged 6 days later without major complications.

Nine months after surgery, the patient was introduced to our clinic for second opinion complaining of pain in his left hip and difficulty walking. We recognized evident "intoe" walking with patellofemoral and groin pain. Clinical examination revealed an increase in internal rotation to $90^{\circ}$ with reduced external rotation to $10^{\circ}$. Radiological examination revealed fracture consolidation with signs of femoral malrotation. Suspecting a rotational malunion, the study was completed with a computed tomography (CT) in order to measure her femoral anteversion.

\section{Rotational malalignment measurement}

CT images were treated with a $3 \mathrm{D}$ reconstruction software 

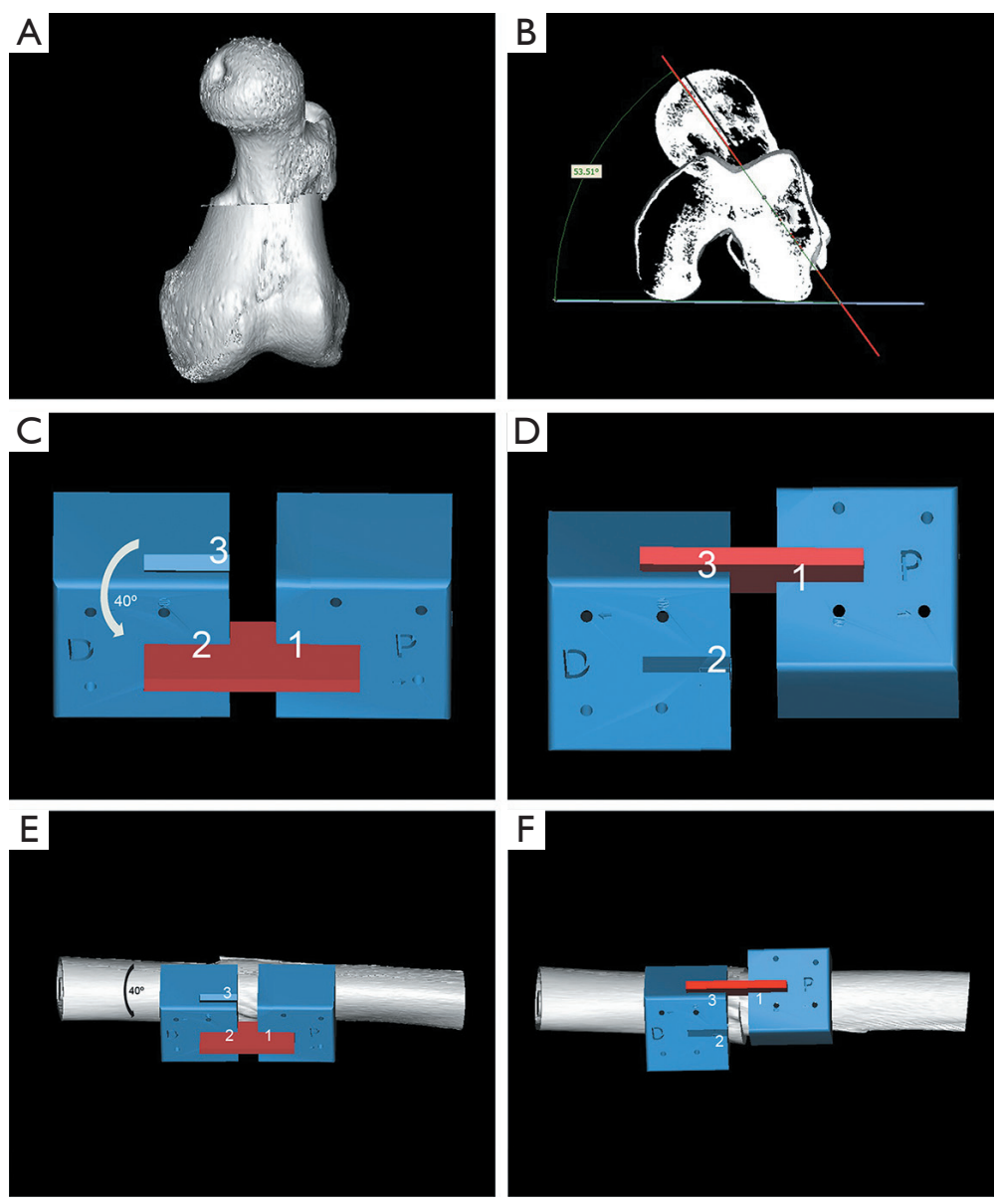

Figure $13 \mathrm{D}$ surgical planning. (A) A 3D composition of the femoral head and condyles is performed from CT images of the patient; (B) thus, the angle of femoral anteversion is determined more realistically and accurately; (C,D) the guide consisted of two pieces (distal and proximal) between which there was a space for cutting bone during osteotomy. Both pieces provided with holes for temporary bone fixation by monocortical screws or wires. The proximal part presented one slot [1] and the distal part had two slots [2,3], in which an auxiliary piece (red) was placed to join both pieces; (C,E) the union of the two parts through the slots number 1 and number 2 adapted the guide to femur at its initial position; $(\mathrm{D}, \mathrm{F})$ the guide was designed so that by rotating the distal femoral part and aligning both pieces through slots 1 and 3 , a bone external rotation of $40^{\circ}$ occurred, necessary for the correct alignment of the femur.

(Mimics ${ }^{\circledR}$, Materialise, Belgium), obtaining a 3D composition of femoral head and condyles (Figure $1 A$ ). The femoral rotational malalignment was accurately measuring by the software according to the technique described by Jeanmart (1), which determines the angle between a line tangential to the dorsal bony contours of the femoral condyles and a line drawn through the axis of the femoral neck (Figure 1B). This analysis showed a femoral anteversion angle of $53.51^{\circ}$ on the injured leg compared to the healthy side, with an angle of $13^{\circ}$. Therefore, a difference of $40^{\circ}$ was found between the two legs, causing an increased internal rotation.

\section{D planning}

After quantifying the degree of correction needed, preoperative planning was performed. A diaphyseal derotational osteotomy with intramedullary fixation for correction of excessive femoral anteversion was considered in order to carry out a new osteosynthesis in the focus of previous fracture. To date, surgical techniques for correcting malrotation are observer-dependent, based on 

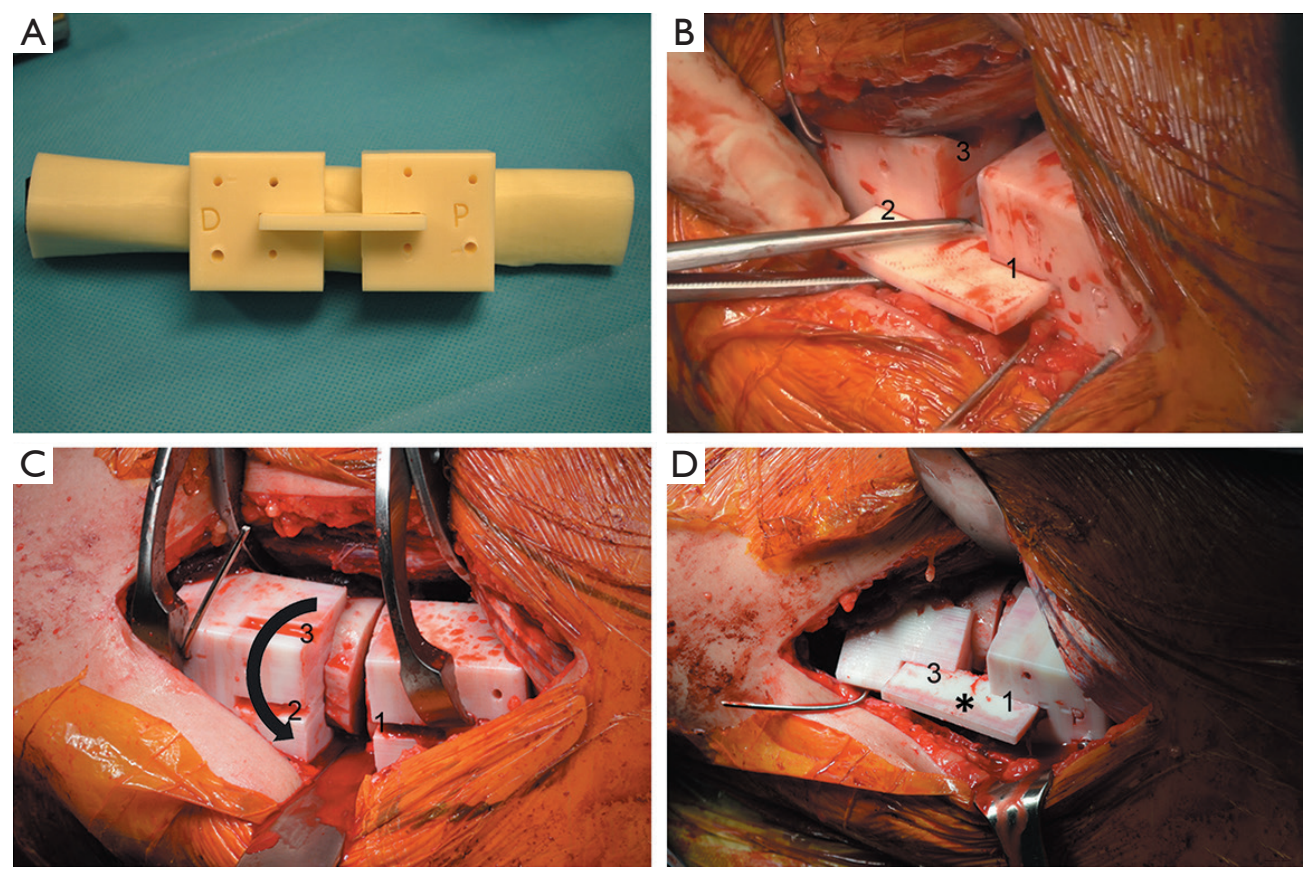

Figure 2 Surgical procedure. (A) Surgical guide and femur of the patient were 3D printed to check their adjustment; (B) surgical guide was fixed to the bone at its initial position aligning slots 1 and 2; (C) after performing the femur osteotomy through space between the guide parts, a distal femoral rotation is performed to align the slots 1 and 3; (D) the correct alignment is checked and secured by placing the auxiliary part (asterisk).

measurements of intraoperative clinical and radiological parameters. Our goal was to create a surgical guide that would control more accurately the correcting femoral rotation.

First, 3D femur reconstruction was achieved from CT images. Next, an adapted guide to femur bone surface was designed to direct the rotation of bone fragments exactly without radiological control after performing the femoral cut (Figure 1C-F) (Autodesk Invertor Professional, Autodesk, USA). Also it would bring temporary stability after osteotomy, facilitating the new intramedullary nailing. Finally, a virtual simulation of osteotomy as well as the use of intraoperative guide was conducted.

\section{Surgical technique}

Once the result of the computer simulation was successful, the piece was printed in a $3 \mathrm{D}$ printer using $\mathrm{ABSplus}$ plastic (Dimension sst $1200 \mathrm{es}$, Stratasys, USA), and sterilized for utilizing during surgery (Figure 2A). Previous T2 nail was extracted from greater trochanter. Subsequently, the femoral diaphysis was exposed and the surgical guide was fixed to bone with two monocortical wires for each piece in order to achieve a correct fixation at its initial position (Figure 2B). After conducting femur osteotomy through the gap between the two pieces of the guide, correction of $40^{\circ}$ of excessive femoral anteversion was performed with an external rotation of the distal femoral part (Figure $2 C, D$ ). Next, it was introduced a new T2 nail with proximal dynamic locking and distal locking. As the guide wires were monocortical, it was not necessary to remove the guide to put the new nail, maintaining the correction and providing stability for the intervention. Once nail was placed, surgical guide was removed and correct positioning was checked under fluoroscopy. One month after surgery, the patient presented normal walking, with internal rotation at $90^{\circ}$ and external rotation at $50^{\circ}$, similar to contralateral leg. She presented no pain in hip and knee.

\section{Discussion}

The gold standard technique for treating femoral shaft 
fractures in adults is the placement of intramedullary nails. This technique provides high fracture healing and allows early mobilization (2). However, misalignment during surgery can lead to femoral malrotation, which has been reported in $28 \%$ of cases (3). This malrotation, defined as a difference of more than $10^{\circ}-15^{\circ}$ between the affected leg and healthy side, can lead to problems in the hip, knee and patellofemoral joint as well as futures osteoarthritic problems (4). Achieving real and accurate measurements of malrotation is necessary to both the diagnosis and subsequent correction.

There are different methods to analyze the femoral anteversion based on clinical examination, fluoroscopy, ultrasonography or CT scan (5). The latter offers more accuracy, measuring the angle between the line through the center of the femoral neck and tangential line to the posterior side of femoral condyles (1). The major complication of this technique is the correct position and orientation of the lines to form the angle, which may be inadequate since employing $2 \mathrm{D}$ models can distort the patient's anatomy (6). In this case, we improved this analysis by achieving a 3D model from CT, recreating the exact femoral anatomy and reducing the error in the anteversion assessment.

During the femoral derotation osteotomy and placement of the intramedullary nail, errors may also occur when the desired rotation degrees have to be achieved (7). To avoid this, we designed a custom surgical guide that indicated the needed rotation of the femoral distal part for proper alignment. 3D technology allowed simulating and validating this process before the surgery intervention. By a $3 \mathrm{D}$ printer, we achieved in a short time and with low cost this surgical guide, able to fully adapt to the patient's bone, and its employ during the intervention led to successful alignment and with minimal use of fluoroscopy.

This case exemplifies that the use of 3D technology and printing (8-10), from diagnosis to treatment, can help to achieve successful results in a rapid and economical manner, reducing intervention times and minimizing risks to the patient.

\section{Acknowledgements}

None.

\section{Footnote}

Conflicts of Interest: The authors have no conflicts of interest to declare.

Informed Consent: Written informed consent was obtained from the patient for publication of this manuscript and any accompanying images.

\section{References}

1. Jeanmart L, Baert A, Wackenheim A. Computed Tomography of Neck, Chest, Spine and Limbs. New York: Springer, 1983;3:171-7.

2. Winquist RA, Hansen ST Jr, Clawson DK. Closed intramedullary nailing of femoral fractures. A report of five hundred and twenty cases. 1984. J Bone Joint Surg Am 2001;83-A:1912.

3. Jaarsma RL, Pakvis DF, Verdonschot N, et al. Rotational malalignment after intramedullary nailing of femoral fractures. J Orthop Trauma 2004;18:403-9.

4. Karaman O, Ayhan E, Kesmezacar H, et al. Rotational malalignment after closed intramedullary nailing of femoral shaft fractures and its influence on daily life. Eur J Orthop Surg Traumatol 2014;24:1243-7.

5. Lindsey JD, Krieg JC. Femoral malrotation following intramedullary nail fixation. J Am Acad Orthop Surg 2011;19:17-26.

6. Murphy SB, Simon SR, Kijewski PK, et al. Femoral anteversion. J Bone Joint Surg Am 1987;69:1169-76.

7. Jaarsma RL, van Kampen A. Rotational malalignment after fractures of the femur. J Bone Joint Surg Br 2004;86:1100-4.

8. Jeong HS, Park KJ, Kil KM, et al. Minimally invasive plate osteosynthesis using $3 \mathrm{D}$ printing for shaft fractures of clavicles: technical note. Arch Orthop Trauma Surg 2014;134:1551-5.

9. Cai H. Application of 3D printing in orthopedics: status quo and opportunities in China. Ann Transl Med 2015;3:S12.

10. Wu XB, Wang JQ, Zhao CP, et al. Printed threedimensional anatomic templates for virtual preoperative planning before reconstruction of old pelvic injuries: initial results. Chin Med J (Engl) 2015;128:477-82.

Cite this article as: Fiz N, Delgado D, Sánchez X, Sánchez P, Bilbao AM, Oraa J, Sánchez M. Application of 3D technology and printing for femoral derotation osteotomy: case and technical report. Ann Transl Med 2017;5(20):400. doi: 10.21037/ atm.2017.07.03 